Published in final edited form as:

Nat Hum Behav. 2018 November ; 2(11): 797-799. doi:10.1038/s41562-018-0471-8.

\title{
Using Rigorous Methods to Advance Behaviour Change Science
}

\author{
Jennifer A. Sumner ${ }^{a,}{ }^{,}$, Rachel N. Carey ${ }^{b}$, Susan Michie ${ }^{b}$, Marie Johnstonc, Donald \\ Edmondson ${ }^{a}$, and Karina W. Davidson ${ }^{a}$ \\ aCenter for Behavioral Cardiovascular Health, Columbia University Medical Center, New York, NY, \\ USA \\ bDepartment of Clinical, Educational, and Health Psychology, Centre for Behaviour Change, \\ University College London, London, UK \\ Institute of Applied Health Sciences, University of Aberdeen, Aberdeen, UK
}

\begin{abstract}
The burden of disability-adjusted life years, prevalence of chronic health conditions, and existence of other global challenges such as pollution are common, costly, and often preventable. ${ }^{1,2}$ Behaviors are critical targets of intervention to improve physical and mental well-being, longevity, and the environment. ${ }^{2}$ However, the science behind, and findings of, behavior change interventions are frequently fragmented and inconsistently reported, and interventions are often unsuccessful in initiating and/or sustaining behavior change. ${ }^{3,4}$ As a result of fragmentation and poor reporting, much research wastes opportunities to build forward momentum, limiting opportunities to harness and synthesize findings to systematically improve behavior change interventions, and the science informing them. ${ }^{5}$ Here, we describe two recent initiatives on behavior change—one from the United Kingdom (UK) and one from the United States (US) — that were developed specifically, albeit independently, to tackle these problems. Although by no means the only international behavior change initiatives, we highlight them here because they both aim to advance behavior change science through an emphasis on common, systematic, and rigorous methods, and they take different, but complementary, approaches in this pursuit. We believe that uniting these and other initiatives will cultivate a more cumulative science of behavior change that can inform policy, practice, and scientific progress.
\end{abstract}

\section{The UK Initiative on Behavior Change}

Funded by the Wellcome Trust and led by researchers at University College London, the Universities of Aberdeen and Cambridge, and IBM Dublin, the Human Behaviour-Change Project (HBCP) is a collaboration of behavioral scientists, computer scientists, and system

\footnotetext{
*Corresponding author: Jennifer A. Sumner, Center for Behavioral Cardiovascular Health, Colubia University Medical Center, 622 W. $168^{\text {th }}$ St, PH9-322, New York, NY 10032, USA. Tel: 1-212-342-3133, Fax: 1-212-342-3431, js4456@ cumc.columbia.edu.

Author Contributions

JAS and KWD led the drafting of the paper. All authors contributed to the manuscript, commented on successive drafts, and read and approved the final version.

Competing Interests

Susan Michie (Principal Investigator), Marie Johnston (Co-Investigator), and Rachel N. Carey are members of the Human BehaviourChange Project. Karina W. Davidson (Co-Principal Investigator), Donald Edmondson (Co-Principal Investigator), and Jennifer A. Sumner (Co-Investigator) are members of the Resource and Coordinating Center for the Science of Behavior Change Research Network.
} 
architects (www.humanbehaviourchange.org). ${ }^{4} \mathrm{HBCP}$ is developing a method and user interface to enable policy makers, practitioners, and researchers to answer variants of the "big question" in behavioral science: What behavior change interventions work, compared with what, for what behaviors, how well, for how long, for whom, in what setting, and why? $\mathrm{HBCP}$ researchers are harnessing advances in behavioral and information science to build a Behavior Change Intervention Ontology (BCIO) and interactive Knowledge System to organize the existing and accelerating evidence about behavior change interventions. ${ }^{4}$ The BCIO aims to establish a controlled vocabulary and connections between behavior change techniques and behavioral outcomes via underlying mechanisms of action, while taking into consideration moderation by intervention exposure (reach and engagement) and context (population and setting). Developing this ontology entails identifying key entities and their definitions, and the relationships between entities. Lower-level ontologies are being developed for each of its entities as well, such as the Behaviour Change Technique Taxonomy (www.ucl.ac.uk/behaviour-change-techniques). ${ }^{6}$

Initially, the Knowledge System database will be populated through manual annotation of behavior change intervention studies (e.g., identifying parts of text, tables, and/or figures from a study and associating them with BCIO entities). Ultimately, artificial intelligence (AI), natural language processing, and machine learning methods will automatically extract this information, in addition to synthesizing and interpreting evidence in a way that gives upto-date answers to commonly asked questions (Figure 1a). ${ }^{4}$ A Paper Authoring Tool is being developed in association with $\mathrm{HBCP}$ to promote uniform reporting of BCIO entities in behavior change intervention publications. This will, in turn, facilitate annotation and population of the database. HBCP is benefiting from prior work to advance standardized research reporting, such as that of the Open Biological and Biomedical Ontology Foundry ${ }^{7}$ and Core Outcome Measures for Effectiveness Trials Initiative. ${ }^{8}$

The Knowledge System with AI, natural language processing, and machine learning methods aims to generate new insights about behavior change from existing evidence and provide explanations of how insights were derived. For example, a research team might query the Knowledge System to access the evidence for the effectiveness of an intervention for behavior change that it plans to incorporate into a study ("What is the evidence for the effectiveness and mechanisms of action of interventions for promoting physical activity in the workplace?"), whereas policy makers planning a public health messaging campaign might query whether certain contextual factors (e.g., population, setting) impact whether such an intervention is successful ("Are there contextual factors that moderate the effect of smoking cessation media campaigns on quitting behavior?”). Currently, HBCP is working with randomized controlled trials used in meta-analyses of Cochrane reviews, with the first use case being the target behavior of smoking cessation. Ultimately, HBCP aims to synthesize all published evaluation evidence relevant to a particular question and present the evidence in accessible forms. While it will have the capacity to make inferences from what is known to what is not yet known, its contribution is limited by the quality of the primary research. 


\section{The US Initiative on Behavior Change}

The Science of Behavior Change (SOBC) program seeks to make behavior change research more impactful, targeted, and systematic by promoting a common, mechanism-focused, experimental medicine approach (www.scienceofbehaviorchange.org). ${ }^{3}$ Even when an intervention results in behavior change, we rarely know how the intervention succeeded. SOBC aims to address this scientific problem by opening the "black box" of behavior change and shedding light on underlying basic behavior change mechanisms. Supported by the National Institutes of Health Common Fund, eight research teams across the US, along with a Resource and Coordinating Center at Columbia University Medical Center, are working to accomplish this goal. ${ }^{3}$ To identify key mechanisms of behavior change, SOBC's experimental medicine approach involves 1) identifying a hypothesized mechanism of behavior change, 2) measuring the mechanism in reliable and valid ways, 3) manipulating that mechanism experimentally, and then 4) determining whether engaging the mechanism results in behavior change (Figure 1b). ${ }^{3,9}$ This initiative aims to develop a common method that is systematic, rigorous, and transparent. By using a standardized scientific approach, SOBC overcomes some of the problems resulting from the use of disparate, nonsystematic approaches to study, and intervene on, behavior change.

SOBC is providing researchers with measures of behavior change mechanisms that are systematically validated. ${ }^{3}$ SOBC researchers are applying the experimental medicine approach to behavior change mechanisms related to self-regulation, stress reactivity and resilience, and interpersonal and social processes. ${ }^{3}$ Measures of these mechanisms currently being validated based on the SOBC method are curated as part of the SOBC Measures Repository (www.scienceofbehaviorchange.org/measures). If researchers start using a standardized set of measures across different interventions, it will be possible to have a harmonized domain of information about how behavior change interventions work, for whom, and under what circumstances. The SOBC Measures Repository allows researchers to locate appropriate measures for their hypothesized mechanism of action and to systematically add to the evidence base regarding whether their behavior change intervention worked as intended. Ultimately, the aim is for knowledge from SOBC to be used to inform the development of more efficient and effective interventions that engage these mechanisms. Uptake of the SOBC method and validated measures has the potential to produce a more coordinated, cumulative science of behavior change.

\section{Different Approaches}

$\mathrm{HBCP}$ is focused on evidence synthesis, integration, and hypothesis generation by harnessing the BCIO and Knowledge System, whereas SOBC is focused on creation of new evidence, use of a common experimental medicine approach, and standardization of target identification, measurement, engagement, and influence on behavior change. Thus, $\mathrm{HBCP}$ aims to enhance research outputs by making connections among studies and inferring beyond them, and SOBC aims to enhance research inputs by creating a standard method to use in new studies and to test if key behavioral mechanisms are influenced in future behavior change intervention studies. While both initiatives include mechanisms of action underlying behavior change in the evidence they are creating and curating, they differ in scope. HBCP 
features broad coverage of mechanisms of action, starting with those listed in 83 theories of behavior change identified in a systematic review and in 14 domains of behavior change identified by expert consensus. In contrast, SOBC's approach employs a narrow and indepth investigation of a few mechanism domains: self-regulation, stress reactivity and resilience, and interpersonal and social processes.

\section{How Can We Harness Both Initiatives For Greater Impact?}

Given these differences in approach, how can we reconcile these initiatives and harness their strengths for greater impact? While SOBC strives to produce better evidence, HBCP strives to make best use of the evidence we have. In the long term, these two approaches are working towards the same goal: a fully accessible, real-time evidence base constructed on high-quality research designs and measurement. $\mathrm{HBCP}$ and SOBC are striving to add structure and cohesion to a field whose knowledge can be scattered, and they are developing collaborative, systematic, fully and openly reported methods and outputs that can be used to advance behavior change science. Further, both initiatives produce tangible resources for researchers, with HBCP building online resources such as the Theories and Techniques Heatmap Tool (www.theoryandtechniquetool.humanbehaviourchange.org/) and SOBC generating the Measures Repository (www.scienceofbehaviorchange.org/measures). By providing common, rigorous, and transparent frameworks and resources for investigators, and by drawing on $\mathrm{AI}$ and other computational methods, these initiatives aim to enable the detection of patterns within observed variations of methodology, theories, and empirical findings. This can, in turn, support the generation of new hypotheses and promote a more efficient, cumulative science of behavior change, leading to accelerated scientific progress.

The work of HBCP and SOBC are informing one another in a variety of ways. For example, HBCP outputs indicating which behavior change techniques are linked to which mechanisms of action are being used by SOBC researchers to identify where they should "go deep" and apply their experimental medicine approach to develop validated measures of promising mechanisms and test these in studies of behavior change. The SOBC "narrow and deep" generation of new evidence on behavior change mechanisms will be guided by the evidence syntheses and inferences of HBCP, and the HBCP Knowledge System will benefit from the evidence generated by SOBC. Together, these approaches aim to create a more refined, useful, and accessible evidence base (Figure 2).

\section{A Call to Action for Advancing Behavior Change Science}

The field of behavior change science requires a generation of researchers who are wellversed in rigorous, methods-focused approaches to behavior change. To produce a systemic shift in how behavior change science is conducted and synthesized, we need scientists who can extend the work described here by engaging in systematic, rigorous behavior change research that routinely tests behavior change techniques and mechanisms of action.

Requiring the use of these new methods and resources in calls for proposals from research funding agencies - something already seen in recent National Institutes of Health funding opportunities—will also promote widespread uptake. 
We are in an exciting age of behavior change research, and the scientific community is in a prime position to creatively disrupt the scientific methods and approaches employed in this field. Researchers around the world are connecting more than ever before across disciplines and countries, recognizing the limitations of individual research groups and single methods, and widely sharing resources. $\mathrm{HBCP}$ and SOBC conduct joint workshops and symposia at conferences worldwide to train researchers in these systematic and transparent approaches to behavior change science, and they collaborate with other related international initiatives, such as the International Behavioural Trials Network (www.ibtnetwork.org) and Stress Measurement Network (www.stresscenter.ucsf.edu). This provides opportunities for broad engagement with new methods and resources, and for promoting collaboration among a global scientific community. By linking the methods and resources of the two initiatives presented here with others, including those just emerging, the science of behavior change can be transformed in ways that make lasting improvements for society.

\section{Acknowledgements}

This work was supported by the National Institutes of Health (NIH) Science of Behavior Change Common Fund Program through an award administered by the National Institute on Aging (U24AG052175) and by a Wellcome Trust collaborative award (The Human Behaviour-Change Project: Building the science of behaviour change for complex intervention development, 201,524/Z/16/Z).

\section{References}

1. Murray CJ, et al. Disability-adjusted life years (DALYs) for 291 diseases and injuries in 21 regions, 1990-2010: a systematic analysis for the Global Burden of Disease Study 2010. The Lancet 380, 2197-2223 (2012).

2. World Health Organization. Global health risks: mortality and burden of disease attributable to selected major risks. (World Health Organization, 2009).

3. Nielsen L, et al. The NIH Science of Behavior Change Program: transforming the science through a focus on mechanisms of change. Behaviour Research and Therapy 101, 3-11 (2018). [PubMed: 29110885]

4. Michie S, et al. The Human Behaviour-Change Project: harnessing the power of artificial intelligence and machine learning for evidence synthesis and interpretation. Implementation Science 12, 121 (2017). [PubMed: 29047393]

5. Glasziou P, et al. Reducing waste from incomplete or unusable reports of biomedical research. The Lancet 383, 267-276 (2014).

6. Larsen KR, et al. Behavior change interventions: the potential of ontologies for advancing science and practice. Journal of Behavioral Medicine 40, 6-22 (2017). [PubMed: 27481101]

7. Smith B, et al. The OBO Foundry: coordinated evolution of ontologies to support biomedical data integration. Nature Biotechnology 25, 1251-1255 (2007).

8. Williamson PR, et al. The COMET Handbook: version 1.0. Trials 20, 280 (2017).

9. Sheeran P, Klein WM, \& Rothman AJ Health behavior change: moving from observation to intervention. Annual Review of Psychology 68, 573-600 (2017). 


\section{Standfirst}

The field of behaviour change suffers from significant fragmentation and poor reporting. Here, we describe two large-scale initiatives-the Human Behaviour-Change Project and Science of Behavior Change program-that aim to introduce complementary systematic and rigorous methods to advance the science of behaviour change. 
a)

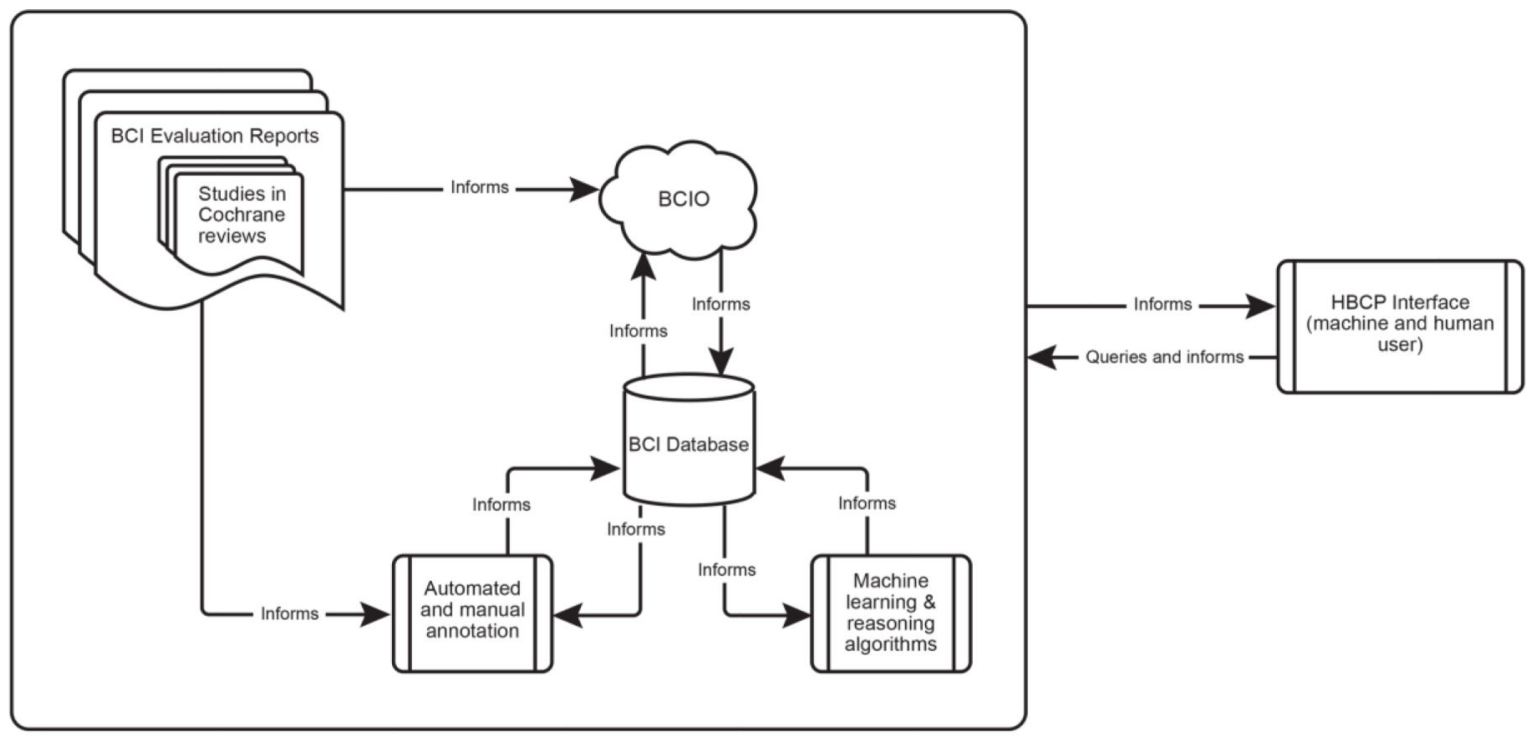

b)

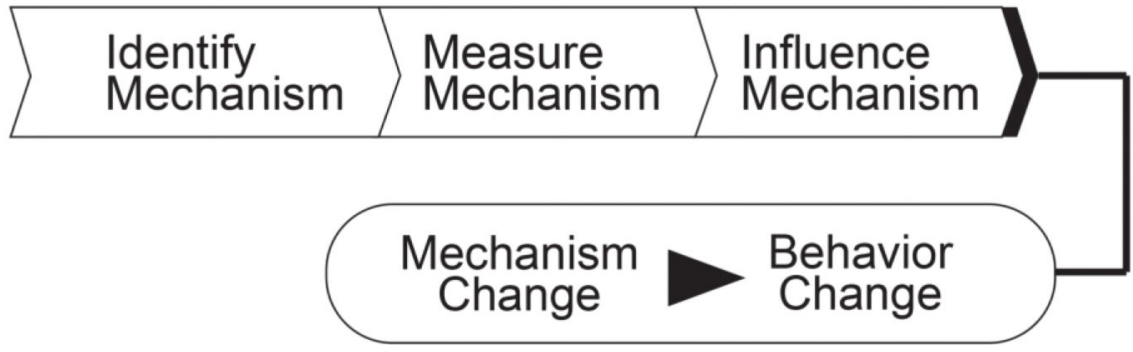

Figure 1. Examples of the Human Behaviour-Change Project (HBCP) and Science of Behavior Change (SOBC) approaches to behavior change science.

Figure 1a presents components of the HBCP Knowledge System for generating new evidence about behaviour change interventions (BCIs). Schematic relationships between the BCI literature, the Behaviour Change Intervention Ontology (BCIO), and a machine and human user interface are depicted [Michie, S., et al. The Human Behaviour-Change Project: harnessing the power of artificial intelligence and machine learning for evidence synthesis and interpretation. Implementation Science 12, 121 (2017)]. Figure 1b presents the steps of the SOBC experimental medicine approach. This method is used to identify key mechanisms underlying behavior change. 


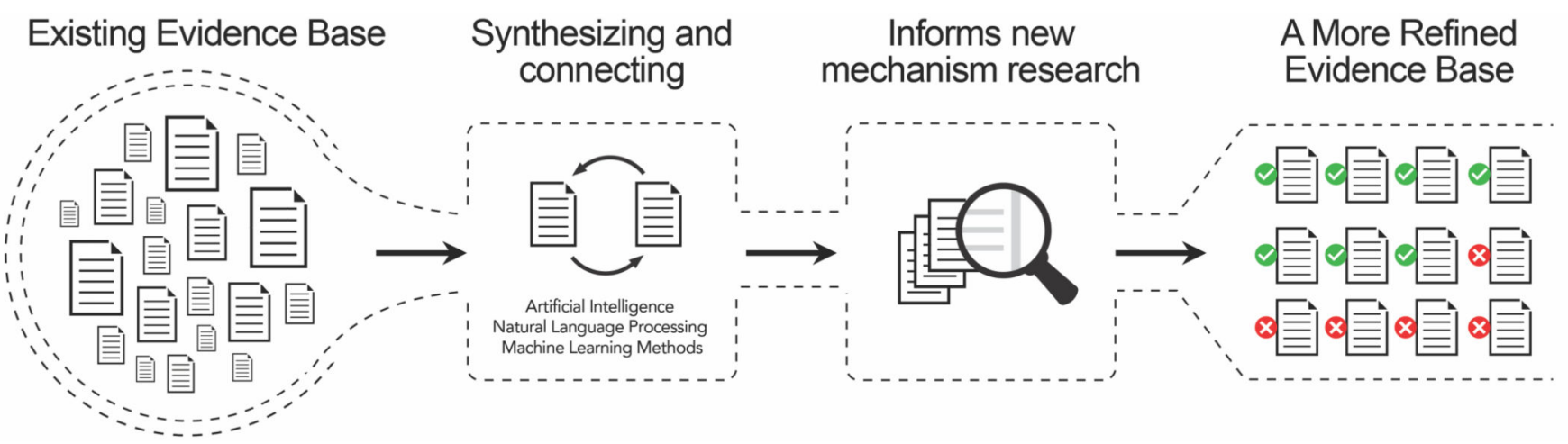

Figure 2. How the approaches of the Human Behaviour-Change Project (HBCP) and Science of Behavior Change (SOBC) can advance behavior change research.

Using artificial intelligence, natural language processing and machine learning, the HBCP aims to organize the fragmented and nonsystematic existing literature in ways that can generate new, accessible evidence. These outputs can show the field where to "go deep" and further examine mechanisms underlying behavior change in new research using rigorous and systematic methods, like the SOBC experimental medicine approach. These additions to the empirical literature, with further processing with artificial intelligence, natural language processing, and machine learning methods, will further refine the evidence base. 